\title{
Erratum: Recharge mixing in a complex distributary spring system in the Missouri Ozarks, USA
}

\author{
Benjamin V. Miller • Robert N. Lerch • \\ Christopher G. Groves • Jason S. Polk
}

Erratum to: Hydrogeology Journal (2014) DOI 10.1007/s10040-014-1225-y

Due to typesetting errors in the prior version, Tables 1 and 2 are reproduced with corrections to data in rows for sites CC, TS2 and TS10 in Table 1, and rows for sites TS13 and WG in Table 2.

Published online: 25 March 2015

(C) Springer-Verlag Berlin Heidelberg 2015

The online version of the original article can be found at http://dx.doi.org/ 10.1007/s10040-014-1225-y.

B. V. Miller $(\bullet) \cdot$ C. G. Groves · J. S. Polk

Hoffman Environmental Research Institute, Department of Geography and Geology, Western Kentucky University, Bowling Green, KY 42101, USA

e-mail: caverben@yahoo.com

B. V. Miller

e-mail: bvmiller@usgs.gov

R. N. Lerch

USDA-Agricultural Research Service, Cropping Systems and Water Quality Research Unit, 1406 Rollins St., Rm. 269, Columbia, MO 65211, USA

Present Address:

B. V. Miller

US Geological Survey, Tennessee Water Science Center, Nashville, TN 37211, USA 
Table 1 Mean ranks and differences among sites for temperature, $p H$, and specific conductivity $(\operatorname{SpC})$ in cool and warm seasons

\begin{tabular}{|c|c|c|c|c|c|c|}
\hline \multirow[b]{2}{*}{ Site } & \multicolumn{2}{|l|}{ Temperature } & \multicolumn{2}{|l|}{$\mathrm{pH}$} & \multicolumn{2}{|l|}{$\mathrm{SpC}$} \\
\hline & $\overline{\mathrm{Cool}}$ & Warm & $\overline{\text { Cool }}$ & Warm & $\overline{\mathrm{Cool}}$ & Warm \\
\hline $\mathrm{CC}^{\mathrm{a}}$ & $1856(\mathrm{~A})^{\mathrm{c}}$ & $786(\mathrm{~F})$ & 1380 (D) & $1829(\mathrm{C})$ & 1193 (D) & $2663(\mathrm{~B})$ \\
\hline TS1 & 1929 (A) & 1650 (D) & $601(\mathrm{~F})$ & 2645 (B) & $367(\mathrm{~F})$ & $677(\mathrm{~F})$ \\
\hline TS2 & 384 (D) & - & 2108 (B) & - & $1653(\mathrm{C})$ & - \\
\hline TS4 & - & 1928 (C) & 一 & 2407 (B) & - & 1850 (D) \\
\hline TS5 & - & $1327(\mathrm{E})$ & - & 957 (E) & - & $888(\mathrm{~F})$ \\
\hline TS6 & - & 2346 (B) & - & 1396 (D) & - & 2199 (C) \\
\hline TS8 & 895 (C) & $755(\mathrm{~F})$ & $1872(\mathrm{C})$ & 1819 (C) & 2122 (B) & 3013 (A) \\
\hline TS10 & $933(\mathrm{C})$ & 2280 (B) & 1502 (D) & $916(\mathrm{EF})$ & 1054 (D) & $810(\mathrm{~F})$ \\
\hline TS11 & 1655 (B) & 1223 (E) & $765(\mathrm{EF})$ & 1583 (CD) & $736(\mathrm{E})$ & $1338(\mathrm{E})$ \\
\hline TS12 & $1846(\mathrm{AB})$ & $879(\mathrm{~F})$ & $195(\mathrm{G})$ & $202(\mathrm{G})$ & $1810(\mathrm{C})$ & 1826 (D) \\
\hline TS13 & 2014 (A) & $1277(\mathrm{E})$ & $830(\mathrm{E})$ & $696(\mathrm{~F})$ & $313(\mathrm{~F})$ & 1973 (CD) \\
\hline WG & 194 (D) & 3035 (A) & 2452 (A) & 3035 (A) & 2457 (A) & $249(\mathrm{G})$ \\
\hline $\mathrm{CD}_{(0.05)}{ }^{\mathrm{b}}$ & 200 & 253 & 200 & 253 & 200 & 253 \\
\hline
\end{tabular}

${ }^{a} C C$ Carroll Cave; $W G$ Wet Glaize Creek

${ }^{\mathrm{b}} \mathrm{CD}_{(0.05)}=$ critical difference value for determining significance between sites; calculated at $\alpha=0.05$

c Mean rank of ratio data relative to reference site TS7. Mean ranks listed for each column, followed by the same letter (in parenthesis) were not significantly different at $\alpha=0.05$

Table 2 Average concentrations of major cations and bicarbonate $\left(\mathrm{HCO}_{3}{ }^{-}\right)$for 11 springs, Carroll Cave $(\mathrm{CC})$, and Wet Glaize Creek $(\mathrm{WG})$

\begin{tabular}{|c|c|c|c|c|c|c|}
\hline Site & $\begin{array}{l}\mathrm{Ca}^{2+} \\
\mathrm{mmol} \mathrm{L}^{-1}\end{array}$ & $\mathrm{Mg}^{2+}$ & $\mathrm{Na}^{+}$ & $\mathrm{K}^{+}$ & $\mathrm{HCO}_{3}^{-}$ & $\begin{array}{l}\mathrm{SpC}^{\mathrm{d}} \\
\mu \mathrm{S} \mathrm{\textrm {cm } ^ { - 1 }}\end{array}$ \\
\hline$\overline{\mathrm{CC}}$ & $0.991 \pm 0.089(\mathrm{AB})^{\mathrm{c}}$ & $1.03 \pm 0.15$ & $0.155 \pm 0.018$ & $0.039 \pm 0.006$ & $4.11 \pm 0.47$ & $364 \pm 54$ \\
\hline TS1 & $0.916 \pm 0.083(\mathrm{ABC})$ & $0.938 \pm 0.123$ & $0.142 \pm 0.020$ & $0.037 \pm 0.006$ & $3.88 \pm 0.39$ & $363 \pm 42$ \\
\hline TS2 & $0.852 \pm 0.078(\mathrm{CD})$ & $0.862 \pm 0.088$ & $0.151 \pm 0.023$ & $0.040 \pm 0.009$ & $3.67 \pm 0.39$ & $343 \pm 38$ \\
\hline TS4 & $0.914 \pm 0.083(\mathrm{ABC})$ & $0.921 \pm 0.113$ & $0.138 \pm 0.017$ & $0.035 \pm 0.006$ & $3.87 \pm 0.39$ & $364 \pm 42$ \\
\hline TS5 & $0.910 \pm 0.073(\mathrm{ABC})$ & $0.919 \pm 0.105$ & $0.140 \pm 0.019$ & $0.037 \pm 0.007$ & $3.84 \pm 0.36$ & $348 \pm 33$ \\
\hline TS6 & $0.925 \pm 0.072(\mathrm{ABC})$ & $0.900 \pm 0.092$ & $0.141 \pm 0.020$ & $0.036 \pm 0.006$ & $3.90 \pm 0.40$ & $349 \pm 35$ \\
\hline TS7 & $0.913 \pm 0.073(\mathrm{ABC})$ & $0.923 \pm 0.101$ & $0.148 \pm 0.025$ & $0.036 \pm 0.005$ & $3.88 \pm 0.38$ & $352 \pm 41$ \\
\hline TS8 & $1.00 \pm 0.09(\mathrm{~A})$ & $0.991 \pm 0.121$ & $0.154 \pm 0.036$ & $0.038 \pm 0.006$ & $4.26 \pm 0.48$ & $396 \pm 45$ \\
\hline TS10 & $0.917 \pm 0.070(\mathrm{ABC})$ & $0.918 \pm 0.108$ & $0.135 \pm 0.018$ & $0.036 \pm 0.005$ & $3.89 \pm 0.40$ & $360 \pm 42$ \\
\hline TS11 & $0.920 \pm 0.077(\mathrm{ABC})$ & $0.921 \pm 0.110$ & $0.140 \pm 0.019$ & $0.036 \pm 0.006$ & $3.87 \pm 0.38$ & $361 \pm 43$ \\
\hline TS12 & $0.966 \pm 0.057(\mathrm{AB})$ & $0.951 \pm 0.125$ & $0.134 \pm 0.021$ & $0.035 \pm 0.007$ & $4.13 \pm 0.30$ & $383 \pm 35$ \\
\hline TS13 & $0.899 \pm 0.086(\mathrm{BC})$ & $0.972 \pm 0.165$ & $0.135 \pm 0.017$ & $0.036 \pm 0.006$ & $3.89 \pm 0.40$ & $363 \pm 42$ \\
\hline WG & $0.802 \pm 0.045$ (D) & $0.856 \pm 0.110$ & $0.154 \pm 0.022$ & $0.044 \pm 0.007$ & $3.69 \pm 0.24$ & $338 \pm 31$ \\
\hline All Sites & $0.917 \pm 0.020$ & $0.931 \pm 0.029$ & $0.144 \pm 0.005$ & $0.037 \pm 0.002$ & $3.91 \pm 0.10$ & $360 \pm 10$ \\
\hline$p$-value ${ }^{\mathrm{a}}$ & 0.013 & 0.663 & 0.846 & 0.772 & 0.615 & 0.717 \\
\hline $\operatorname{LSD}_{(0.05)}{ }^{b}$ & 0.098 & NS & NS & NS & NS & NS \\
\hline
\end{tabular}

${ }^{a} p$-value=probability of observing a more extreme $\mathrm{F}$ statistic from the one-way ANOVA

${ }^{\mathrm{b}} L S D$ least significant difference value calculated at $\alpha=0.05 ; N S$ not significant

${ }^{c}$ mean $\pm 95 \%$ confidence interval; for the $\mathrm{Ca}^{2+}$ data, means in this column followed by the same letter (in parenthesis) were not significantly different at $\alpha=0.05$

${ }^{\mathrm{d}} \mathrm{SpC}=$ specific conductivity 